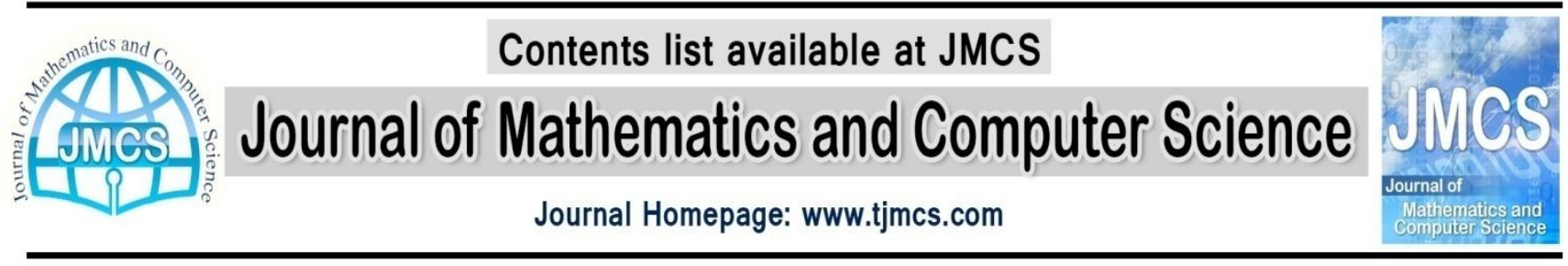

\title{
Computational Model of Social Intelligence Based on Emotional Learning in the Amygdala
}

\author{
Alireza Falahiazar ${ }^{1, *}$, Saeed Setayeshi ${ }^{2}$, Yousef Sharafi ${ }^{1,+}$ \\ ${ }^{1}$ Computer Department of Islamic Azad University Science and Research Branch, Tehran, Iran \\ *a_falahiazar@srbiau.ac.ir \\ +y.sharafi@srbiau.ac.ir
}

${ }^{2}$ Faculty of Nuclear Engineering and Physics, Amirkabir University, Tehran, Iran

setayesh@aut.ac.ir

Article history:

Received July 2014

Accepted October 2014

Available online November 2014

\begin{abstract}
Today, neural systems are being applied as a powerful tool to solve a host of problems. The more computational model of the presented neural system and the neural system of animals look alike, the more powerful the computational model would be. The brain emotional learning algorithm in the Amygdala inspired by Limbic of mammalian brains was put forward originally by Mor'en. A combination of some Limbic systems as some living creatures will be applied in this paper. The gathering of these living creatures and output summation of each Limbic system in accordance with the relevant applied coefficient will result in social intelligence. The proposed model has been compared with feed-forward back propagation network, Elman back propagation network, and a computational model of emotional learning in the Amygdala to forecast Mackey-Glass Chaotic Time Series. The results of the above mentioned comparison concludes that in forecasting Mackey-Glass Chaotic Time Series, the computational model of social intelligence based on the emotional learning in the Amygdala has shown fewer errors in not only training patterns but also testing patterns.
\end{abstract}

Keywords: Machine Intelligence, Social Intelligence, Emotional Learning in the Amygdala, MackeyGlass Chaotic Time Series

\section{Introduction}

Artificial Neural Networks, in a simpler definition Neural Networks, are the new systems and computational methods for machine learning, presenting the knowledge, and eventually applying the obtained knowledge in order that the output responses of the complex systems can be forecasted. The original notion of this type of networks is inspired by the function of biological neural network to process the information and data to be able to learn and create knowledge. The key element of this notion is making new structures for the information processing system. This system is composed of a host of extremely compact processing elements named neurons which work together to coordinate to 
solve a problem. Synapses are connections between neurons through which information flows from one neuron to another. Based on a logical model, McCulloch and Walter Pitts [1] made the first attempts to simulate the neural network which is today the main component of most of artificial neural networks. This model presents some theories about neurons. The performance of this model is based on the summation of inputs and creating outputs. Neurons are so called excited providing that the summation of inputs is more than threshold value. The outcome of this model was the performance of some logical functions such as "AND" and "OR". Not only Neurophysiologists but psychologists and engineers played roles in development of simulating the neural networks. Perceptron network was introduced by Rosenblatt [2] in 1958. This network looked like previous modeled networks. Perceptron includes 3 layers whose 2 nd one is known as link layer. This system can learn that corresponding accidental output should be exerted on the given input. Adaptive linear neuron is another system developed in 1960 by Professor Bernard Widrow and his graduate student Ted Hoff [3] and was the first applied neural system in real problems. In 1969, a book was written by Minsky and Papert [4] in which limitations of one and even multi-layer Perceptron were illustrated. This book caused not only a very poor investment but the demise of research in simulating neural network. They argued that there were certain tasks, which perceptron model could not solve and caused the researches to stop for several years. Despite the fact that public willingness and available investment had almost vanished, a number of researchers pursued the matter further and tried to follow the conducted researches to create the machines which could solve some problems such as pattern recognition. Grossberg [5] introduced a network named avalanche to recognize speech fluency and control of robot hands. Adaptive resonance theory (ART) [6] is also a theory developed by Grossberg and Carpenter which differed from natural models. Anderson [7-9] and Kohonen [10-11] were also the ones who developed some learning techniques. Backward propagation of errors is a common method of training developed by Werbos [12] in 1974 which was a multi-layered Perceptron network with more powerful training rules. The achieved breakthrough from 1970 to 1980 drew a lot of attention to the neural system. Some of the applications of neural networks are for use in stock price prediction [13], bankruptcy Prediction [14], Detect dos-attacks [15] and controlling the False Alarm in an Intrusion Tolerant Database System [16].Neural networks trained with gradient descent based algorithms [17] and evolutionary algorithms [18-19]. A computational model of emotional learning in the Amygdala was initially come forward by Mor'en and Balkenius [20] in 2000. In 2002, Mor'en presented his doctoral thesis [21] on Emotion and Learning. In the second section of the paper, the computational model of emotional learning in the Amygdala will be discussed. The proposed model, computational model of social intelligence based on emotional learning in the Amygdala, will be investigated in the third section. In sections 4 and 5, the implementation outcomes and the conclusions based on the implementation outcomes will be discussed respectively.

\section{The computational model of emotional learning in the Amygdala}

Emotion means movement, incitement, and excitation. Emotion is the brain's various incitements in relation to provocation, spirit, habitude, personality, temper and so forth. In terms of physiology, excitement is caused by tranquillizing hormones or neural stimuli such as Dopamine, Oxytocin, Noradrenaline, Serotonin, and Cortisol [22-23]. Reptiles react to symbols of chemical, touch, and sight senses and their reactions have already been determined in accordance with their bodies. The sense of smell of the mammals which are active at night becomes a dominant sense and replaces the sight sense; consequently, the lobes of sense of smell in mammalian brains in comparison with their sizes are bigger than the reptiles'. Emotion is an organized psychic state in Limbic system of mammalian brains. Emotions are more complicated than reptiles' reflexive responses. Take the emotion of love as an example among the mammals which is developed in Cingulate Gyrus and facilitates the children's nutrition and caring. 
Limbic system can be defined as emotions at specific parts of brain. The most significant parts of this system are Amygdala, Hypothalamic, Cortex Pryfrantal, Gyrus Syngvlat and Hippocampus which are all located in cortex [22]. It should be noted that emotions are not merely related to Limbic system, yet it has been shown that some of these Limbic systems are not directly related to emotions. However, some non-Limbic systems can be found which have more effects on creating emotions.

Emotional computation is considered as an interdisciplinary field including computer science, psychology and cognitive science. However, this field might originally refer to the previous philosophic questions about emotion. Emotional neural network is a field of artificial intelligence which has resulted in designing the systems which can recognize process and even interpret the emotions. Emotion recognition starts with the sensors which receive the raw data about the physical state or user's behavior. The obtained data are compared with the human signs which have already been categorized to understand the emotion. Another improvement in this regard is designing some machines presented to show the innate emotion which are able to simulate the emotions. Analyzing the speech patterns, speech emotional processing recognizes the user's emotional state. Processing is obtained by investigating the facial expressions or the body language through the sensors.

The Amygdala section plays a crucial role in controlling the emotional activities such as friendship, love and kindness, temper, fear, invasion and anger [21]. Amygdala is the center of noticing the perils and is of a great importance for human's survival. The Amygdala section is trained to predict the next reactions and react to the reward. The Orbitofrontal system interferes when the prediction made by the Amygdala and the reward are not consonant with each other. Consequently, based on the former learning, it endeavors to remove this dissonance. The most significant part of learning algorithm is defining the reward function. Reinforced Reward signal is a function of other signals considered as an evaluation function.

The evaluation function evaluates the efficiency of our responses and has a direct connection with the agent's activity and guides the agent towards the main goal, simulation. For instance, rewards and punishments in brain are determined based on the former value of the evaluation function. With a decrease of evaluation function value, reward and punishment will decrease and increase respectively; as a result, responses will change as well. Therefore, Cohesion between the current value of rewards and reactions is one of the aspects of learning, which is the power of increasing the reward by a stimulus to the new learning.

Mo'ren Model [21] is a reduced system of mimicking mammalian brains. Figure 1 illustrates structure of the computational model of Emotional Learning in the Amygdala developed by Mo'ren.

In Forward algorithm [21], the computational model of Emotional Learning in the Amygdala is presented by equations 1-6. Emotional learning algorithm in relevant computational model is presented by equations $7-8[21]$.

$A_{i}=S_{i} V_{i}$

$O_{i}=S_{i} W_{i}$

$E=\sum_{i} A_{i}-\sum_{i} O_{i}$

It should be noted that the final input for the Amygdala section is the maximum of sensory input which is being sent from thalamus to Amygdala whose value is defined in accordance with equation 4.

$$
\begin{aligned}
& A_{t h}=\max \left(S_{i}\right) \\
& A_{i}=S_{i} V_{i} \\
& O_{i}=S_{i} W_{i}
\end{aligned}
$$


$\Delta V_{i}=\alpha \cdot S_{i} \cdot\left(R e w_{v}-\sum_{j} A_{j}\right)$
$\Delta W_{i}=\beta \cdot S_{i} \cdot\left(\sum_{j} A_{j}-\operatorname{Rew}_{w}\right)$

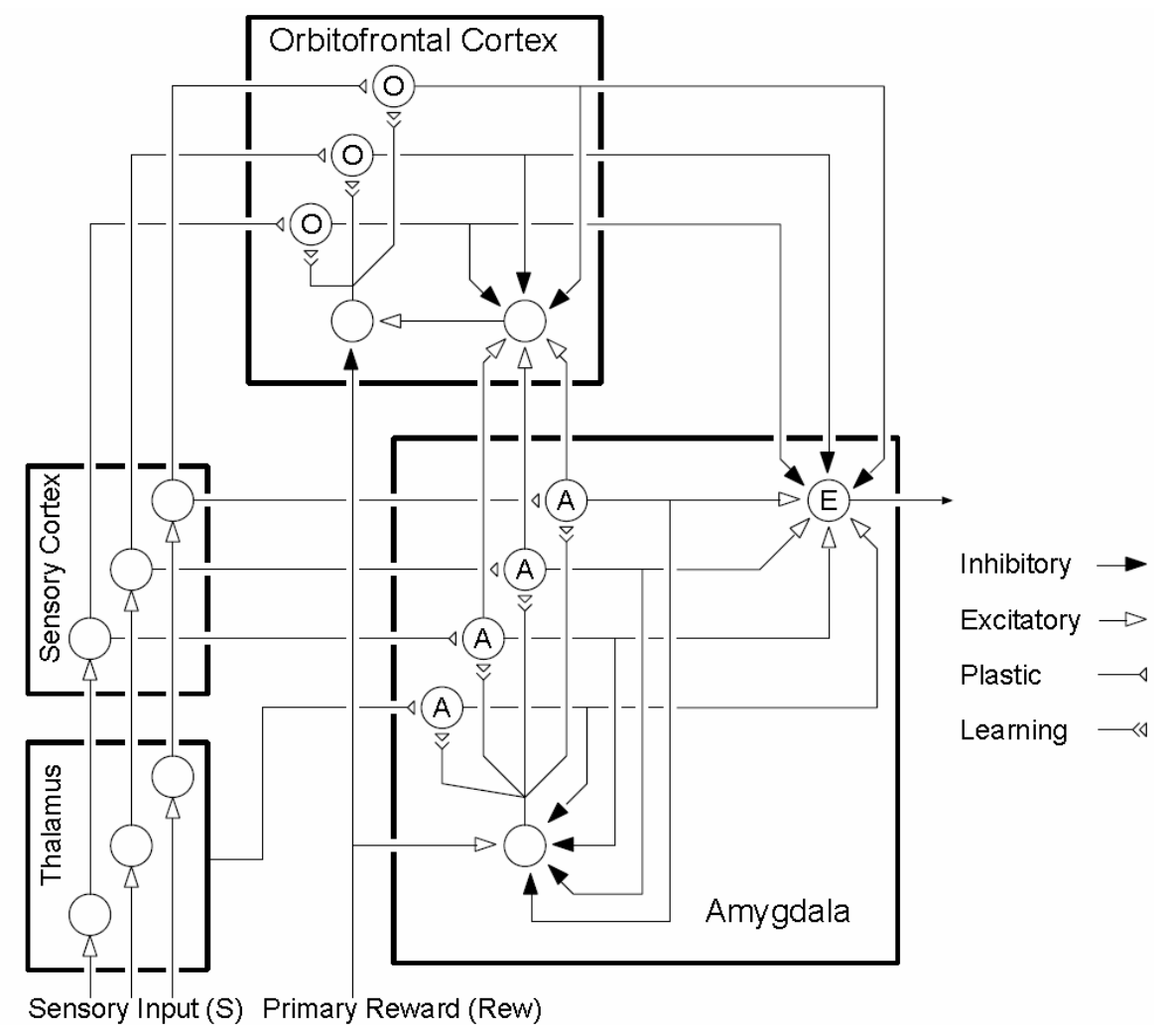

Figure 1. Structure of computational model of Emotional Learning in the Amygdala

The definitions of various important parameters of equations 1-8 are as follow:

- $A_{i}$ : is a set of Amygdala input or in other words, is the result of a multiplication (product) of sensory inputs in $V_{i}$ weights.

- $A_{t h}$ : is the final input of the A input-set to the Amygdala which equals the maximum of the sensory inputs.

- $O_{i}$ : is a set of Amygdala inputs, or in other words is the result of a multiplication (product) of sensory inputs in $W_{i}$ weights in Orbitofrontal.

- $\quad S_{i}$ : is the $i$-th sensory input

- $\quad V_{i}$ : is the $i$-th weight which produces the Amygdala through being multiplied by sensory input in Orbitofrontal.

- $\quad E$ : is the output of Amygdala

- $\operatorname{Rew}_{w}$ : is the reward of updating $V$ weights

- $R e w_{v}$ : is the reward of updating $W$ weights 


\section{The computational model of social intelligence based on emotional learning in Amygdala}

In the proposed model, a Limbic system [21-22] which is the agent of modeled processing in the mammalian brain has been replaced with several ones as the output summation of each Limbic system composes the social intelligence with regard to the relevant coefficient. In actual fact, the decisions made by each living creature are a function of that creature's sensory input or in other words it is its own intelligence plus the intelligence dominated on the environment. In other words, in this social intelligence model, the result of each intelligence is the result of consequence of each creature based on the importance or the applied weights which are adjusted in accordance with the environmental behavior. Based on emotional learning in the Amygdala, computational model of social intelligence is categorized into 2 computational levels, so that the first layer consists of several computational model of emotional learning in the Amygdala. The outputs of the above mentioned models in the second layer are multiplied by the relevant coefficient and then are added together. The final output creates the computational model of social intelligence based on emotional learning in the Amygdala. Figure 2 illustrates structure of computational model of social intelligence based on emotional learning in the Amygdala. The forward algorithm of computational model of social intelligence based on emotional learning in the Amygdala is allocated in equations 9-14. To update the weights of the second layer, back-propagating errors method is used so that equations 15 and 16 will be used to update the second layer. If the back-propagating errors method is used to update the weights of the first layer, the weights of the first layer can be updated by equations 17-20. Since the learning in the first layer which shows a type of emotional training must be according to the equations 7-8, equations 17-20 can be modeled on equations 7-8; consequently, the

gained equations in emotional learning are allocated in equations 21-24. It should be noted that $A_{j}^{i}$ shows the $j$-th weight from the $i$-th computational model of emotional learning.

$$
\begin{aligned}
& \text { Error }=\frac{1}{2} e^{2} \\
& e=\text { desire }-E_{\text {total }} \\
& E_{\text {total }}=\sum_{i} E_{i} W_{i}^{\text {bel }} \\
& E_{i}=\sum_{j} A_{j}^{i}-\sum_{j} O_{j}^{i} \\
& A_{j}^{i}=S_{j} V_{j}^{i} \\
& O_{j}^{i}=S_{j} W_{j}^{i} \\
& W_{i}^{\text {bel }}(k+1)=W_{i}^{\text {bel }}(k)-\gamma \frac{\partial E r r o r}{\partial W_{i}^{\text {bel }}} \\
& \frac{\partial E r r o r}{\partial W_{i}^{\text {bel }}}=\frac{\partial E r r o r}{\partial e} \cdot \frac{\partial e}{\partial E_{\text {total }}} \cdot \frac{\partial E_{\text {total }}}{\partial W_{i}^{\text {bel }}}=e \cdot(-1) \cdot E^{i} \\
& W_{j}^{i}(k+1)=W_{j}^{i}(k)-\beta \frac{\partial E r r o r}{\partial W_{j}^{i}} \\
& \frac{\partial E r r o r}{\partial W_{j}^{i}}=\frac{\partial E r r o r}{\partial e} \cdot \frac{\partial e}{\partial E_{\text {total }}} \cdot \frac{\partial E_{\text {total }}}{\partial E_{i}} \cdot \frac{\partial E_{i}}{\partial O_{j}^{i}} \cdot \frac{\partial O_{j}^{i}}{\partial W_{j}^{i}}=e \cdot(-1) \cdot W_{i}^{b e l} \cdot(-1) \cdot S_{j} \\
& V_{j}^{i}(k+1)=V_{j}^{i}(k)-\alpha \frac{\partial E r r o r}{\partial V_{j}^{i}}
\end{aligned}
$$




$$
\begin{aligned}
& \frac{\partial E r r o r}{\partial V_{j}^{i}}=\frac{\partial E r r o r}{\partial e} \cdot \frac{\partial e}{\partial E_{\text {total }}} \cdot \frac{\partial E_{\text {total }}}{\partial E_{i}} \cdot \frac{\partial E_{i}}{\partial A_{j}^{i}} \cdot \frac{\partial A_{j}^{i}}{\partial V_{j}^{i}}=e \cdot(-1) \cdot W_{i}^{b e l} \cdot(+1) \cdot S_{j} \\
& \Delta V_{j}^{i}=\alpha \cdot S_{j} \cdot\left(\operatorname{Rew}_{v}^{i}-\sum_{k} A_{k}\right) \\
& \operatorname{Rew}_{v}^{i}=\operatorname{desire}+\sum_{j} O_{j}^{i}+W_{i}^{b e l} \\
& \Delta W_{j}^{i}=\beta \cdot S_{j} \cdot\left(\sum_{k} A_{k}-\operatorname{Rew}_{w}^{i}\right) \\
& \operatorname{Rew}_{w}^{i}=\operatorname{desire}-\sum_{j} A_{j}^{i}+2 \sum_{j} O_{j}^{i}+W_{i}^{b e l}
\end{aligned}
$$

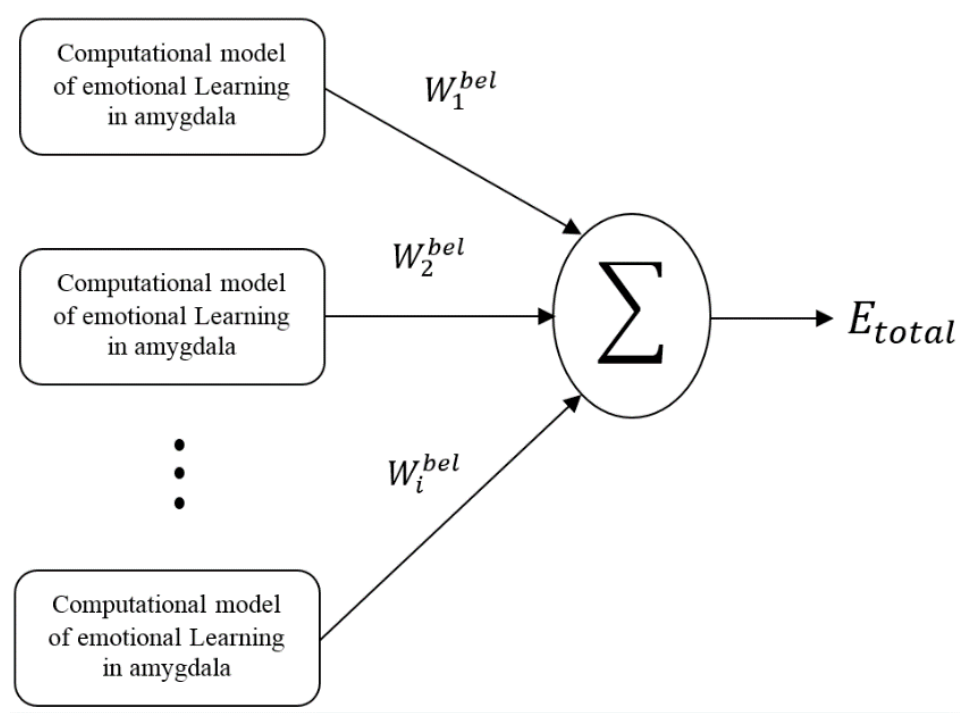

Figure 2. Structure of computational model of social intelligence based on emotional learning in the Amygdala

\section{Implementation results}

The results of the implementation are used as some models such as the computational model of social intelligence based on emotional learning in Amygdala. Feed-forward back propagation network [24] and Elman back propagation network [25] are used to forecast Mackey-Glass Chaotic Time Series [26]. It should be mentioned that the number of used patterns are 1000 so that the producing parameters of Mackey-Glass Chaotic Time Series are adjusted in accordance with equation 25. The first 300 patterns and 700 remained ones are used to train and test the models respectively. 


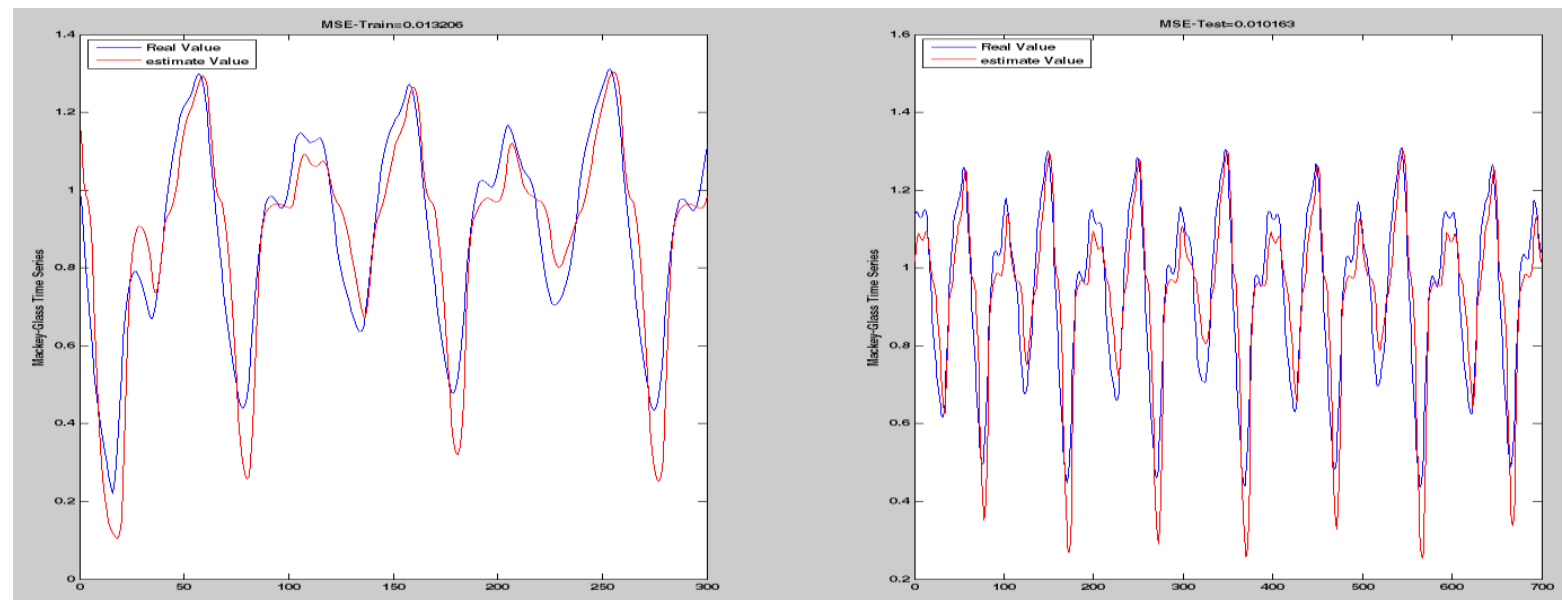

Figure 3. The results of implementing the Feed-forward back propagation network to forecast the Mackey-Glass Chaotic Time Series

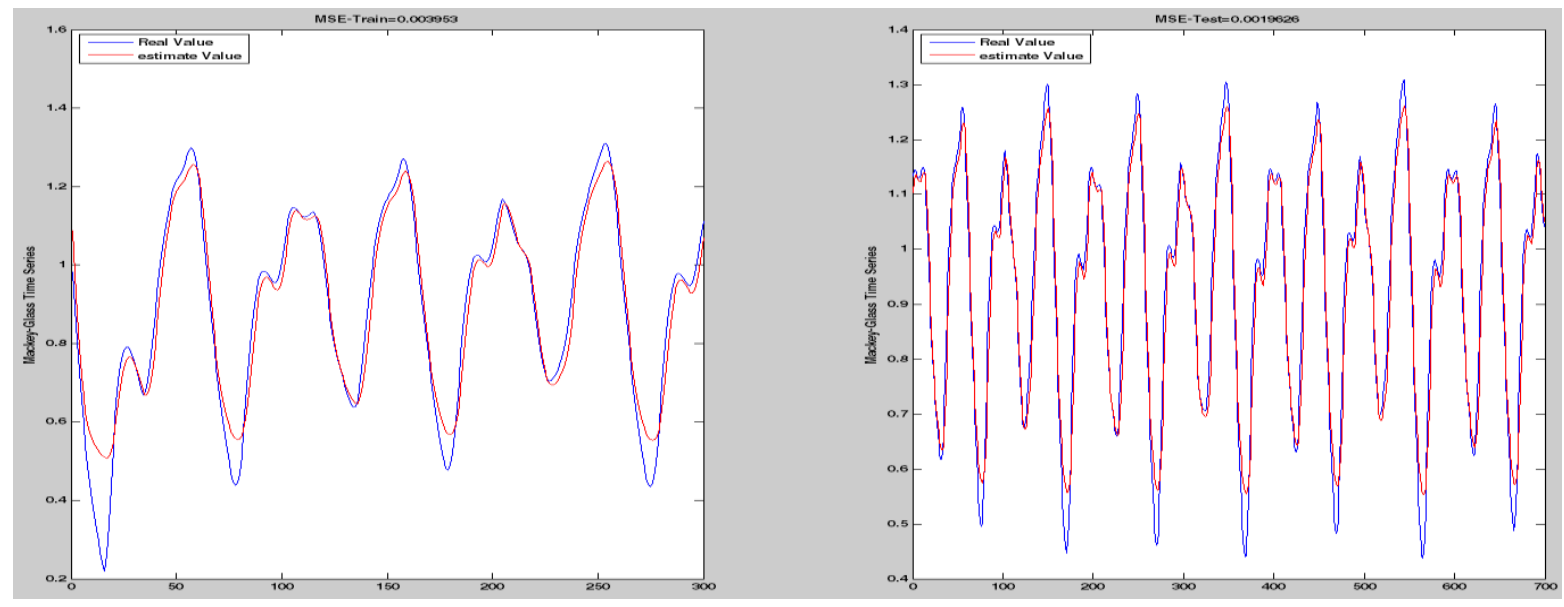

Figure 4. The results of implementing Elman back propagation network to forecast the Mackey-Glass Chaotic Time Series

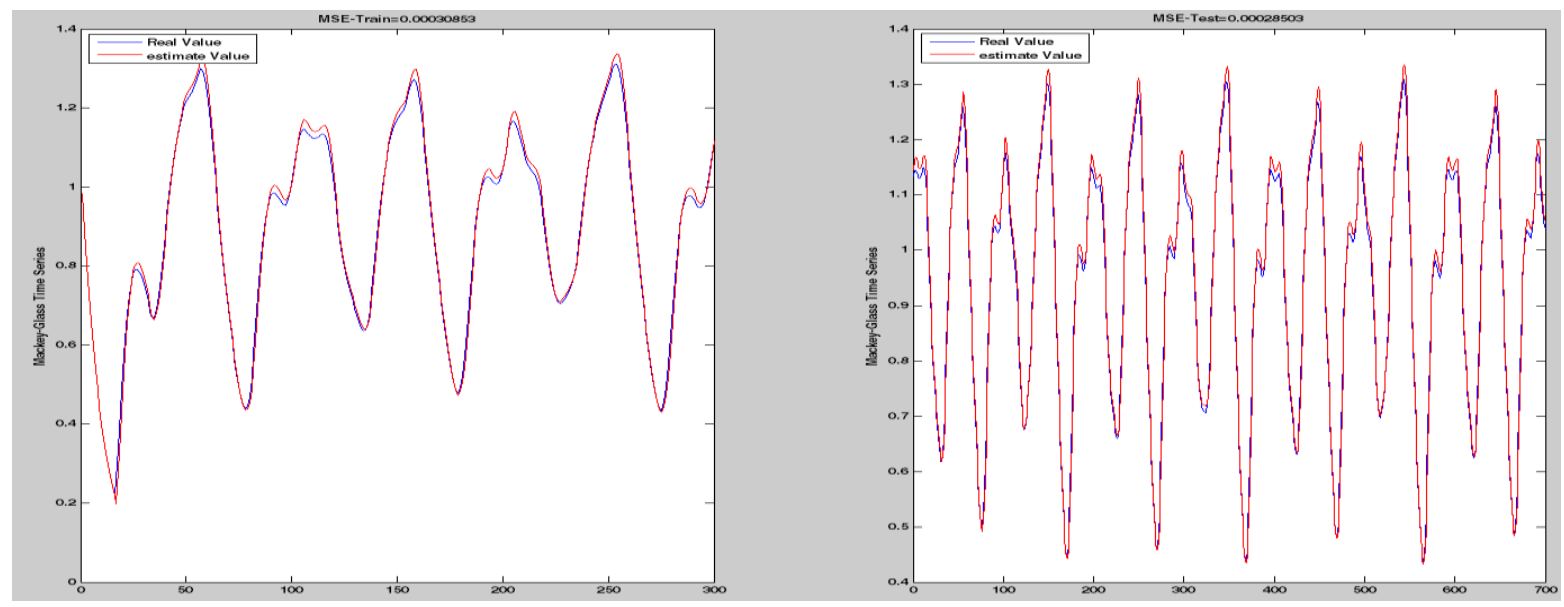

Figure 5. The results of implementing the computational model of emotional learning in the Amygdala to forecast the Mackey-Glass Chaotic Time Series 

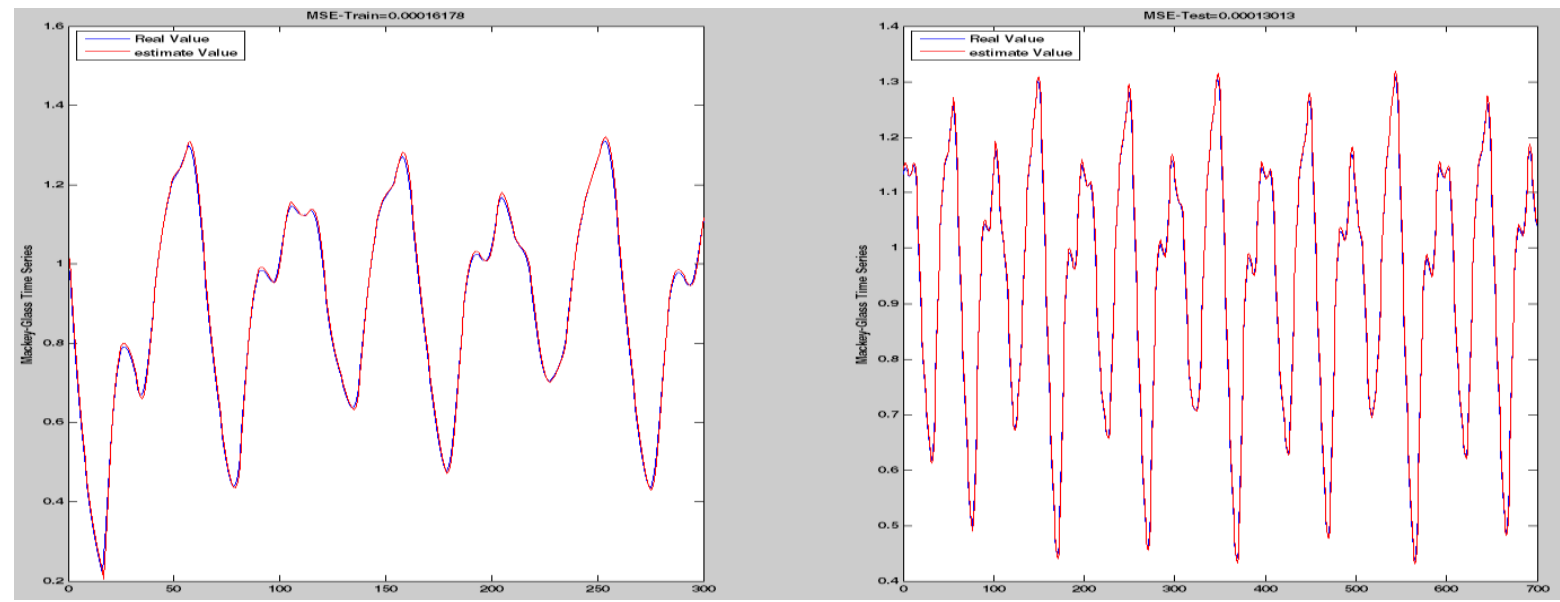

Figure 6. The results of implementing the computational model of social intelligence based on emotional learning in the Amygdala to forecast the Mackey-Glass Chaotic Time Series.

$a=0.2 ; b=0.1 ;$ tau $=17 ; x_{0}=1.2 ;$ delta $=1 ;$ sample $_{n}=1000 ;$ interval $=1$;

To forecast Mackey-Glass Chaotic Time Series in various computational models, two inputs from Mackey-Glass Chaotic Time Series or in other words the value of $\mathrm{i}$-th from the Time series and the value of $(i+1)$-th from time series as the input and the value of $(i+2)$-th from time series as the desired output, where $i=[1, m-2]$ and $m$ can be the number of training patterns or test.

Table 1. The results of implementing of four models to forecast the Mackey-Glass Chaotic Time Series

\begin{tabular}{|l|c|c|c|c|}
\hline & $\begin{array}{c}\text { Feed-forward } \\
\text { back propagation } \\
\text { network }\end{array}$ & $\begin{array}{c}\text { Elman } \\
\text { back propagation } \\
\text { network }\end{array}$ & $\begin{array}{c}\text { emotional } \\
\text { learning in } \\
\text { the Amygdala }\end{array}$ & $\begin{array}{c}\text { social intelligence } \\
\text { based on emotional } \\
\text { learning in the } \\
\text { Amygdala }\end{array}$ \\
\hline $\begin{array}{c}\text { Mean Square Error } \\
\text { for Train Patterns }\end{array}$ & 0.013206 & 0.0003953 & 0.00030853 & 0.00016178 \\
\hline $\begin{array}{c}\text { Mean Square Error } \\
\text { for Test Patterns }\end{array}$ & 0.010163 & 0.0019626 & 0.00028503 & 0.00013013 \\
\hline
\end{tabular}

It should be noted that to train the Feed-forward back propagation network and Elman back propagation network, Gradient descent algorithm is being used. To train in computational model of emotional learning in the Amygdala and computational model of social intelligence based on emotional learning in the Amygdala, emotional learning and a combination of emotional learning and Gradient descent algorithm are used respectively.

The results of implementing the Feed-forward back propagation network, Elman back propagation network, computational model of emotional learning in the Amygdala, and computational model of social intelligence based on emotional learning in the Amygdala to forecast the Mackey-Glass Chaotic Time Series are shown in figures 3-6 respectively. 
It is worth mentioning that in social intelligence of computational model based on emotional learning in Amygdala, 10 limbic systems have been used in the first layer. The mean square error for the mentioned 4 models to forecast the Mackey-Glass Chaotic Time Series is shown in table 1.

\section{Conclusion}

With regard to figures 3-6 and table 1, we will conclude that in comparison with other models, the computational model of social intelligence in the Amygdala has the least mean square error for training and testing patterns. After the computational model of social intelligence in the Amygdala, Elman back propagation network and Feed-forward back propagation network have the least mean square error for training and testing patterns respectively. Consequently, based on the experimental results, the computational model of social intelligence in the Amygdala shows more noticeable results compared with other mentioned models. In Future, to develop the computational model of social intelligence in the Amygdala, the fuzzy systems can be applied so that Orbitofrontal and Amygdala are changed into fuzzy systems.

\section{References}

[1] McCulloch Warren, Walter Pitts, "A Logical Calculus of Ideas Immanent in Nervous Activity". Bulletin of Mathematical Biophysics 5 (4) (1943): 115-133.

[2] Rosenblatt, F., "The Perceptron: A Probalistic Model for Information Storage and Organization In The Brain". Psychological Review 65 (6) (1958): 386-408.

[3] Widrow, B., 6 Stearns, S. D. "Adaptive signal processing. Englewood Cliffs", NJ: Prentice Hall (1985).

[4] Minsky, M., S. Papert, “An Introduction to Computational Geometry”, MIT Press (1969).

[5] Grossberg, S., "Classical and instrumental learning by neural networks", Progress in Theoretical Biology, 3, (1974) pp. 51-141.

[6] G.A. Carpenter, S. Grossberg, "The ART of adaptive pattern recognition by a self-organizing neural network", IEEE Transactions on Computers 21 (3) (1988) 77-88.

[7] Anderson, J.A., Rosenfeld, E., (Eds.), "Neurocomputing: Foundations of Research". Cambridge, MA: MIT Press. Referred to as NFR. [Out of print, available in CompNet Library, (1988).

[8] J.A. Anderson, and S. Lehmkuhle (Eds.), "Synaptic modification, neuron selectivity, and nervous system organization" Hillsdale, NJ: Erlbaum (1985).

[9] Anderson, J.R., "Methodologies for studying human knowledge", Behavioral and Brain Sciences, 10 (1987), 467-505.

[10] T. Kohonen, E. Oja, O. Simula, A. Visa, J. Kangas, "Engineering application of the selforganizing map", Proceedings of the IEEE 84 (10) (1996) 1358-1384.

[11] T. Kohonen, "Self-Organizing Maps", Springer, New York, (1995).

[12] Werbos, P.J., "Beyond Regression: New Tools for Prediction and Analysis in the Behavioral Sciences". (1975).

[13] A. Ghezelbash, "Predicting Changes in Stock Index and Gold Prices to Neural Network Approach," The Journal of Mathematics and Computer Science, vol. 4, no. 2, (2012) pp. 227 236.

[14] M. Bagheri, M. Valipour, V. Amin, "The Bankruptcy Prediction in Tehran share holding using Neural Network and it's Comparison with Logistic Regression", The Journal of Mathematics and Computer Science, vol. 5, no. 3 (2012), pp. 219-228.

[15] M. M. Javidi, M. H. Nattaj, "A New and Quick Method to Detect DoS Attacks by Neural Networks", The Journal of mathematics and computer Science, vol. 6 (2013), pp. 85-96. 
[16] Z. Falahiazar, M. Rohani, A. Falahiazar, "Controlling the False Alarm in an Intrusion Tolerant Database System Using Significance Degrees of Data Objects", Journal of mathematics and computer science, vol. 13 (2014), pp. 212-225.

[17] Hagan, Martin T., Mohammad B. Menhaj. "Training feedforward networks with the Marquardt algorithm", Neural Networks, IEEE Transactions on 5, no. 6 (1994): 989-993.

[18] L. Falahiazar, M. Teshnehlab, A. Falahiazar, "Parallel Genetic Algorithm Based on a New", in International Conference on Recent Advances in Computing and Software Systems, (2012).

[19] Y. Sharafi, M. Ahmadieh Khanesar, M. Teshnehlab, "Discrete Binary Cat Swarm Optimization Algorithm", in Computer, Control \& Communication (IC4), 2013 3rd International Conference on, (2013).

[20] Moren, J., \& Balkenius, C., "A computational model of emotional learning in the amygdala", From animals to animats, 6 (2000), 383-391.

[21] Moren, J., "Emotion and learning a computational model of the Amygdala", Lund University, Vol. 93 (2002).

[22] Friedenberg, J., Silverman, G. "Cognitive science: an introduction to the study of mind", Sage. (2011).

[23] S. Zeki, "The neurobiology of love", in FEBS Lett. Vol. 581 (2007), pp. 2575-2579. PMID 17531984.

[24] Hagan, M. T., Demuth, H. B., Beale, M. H., "Neural network design”, Boston: Pws Vol. 1 (1996).

[25] J. Elman, "Finding structure in time", Cognitive Science, vol. 14 (1990), pp.179 -211.

[26] M. C. Mackey, L. Glass, Science, 197 (1977):287-289. 